\title{
Deep Venous Thrombosis and a Very Rare Finding: Inferior Vena Cava Infra-renal Segment Agenesis
}

\author{
Alexandra Leitão ${ }^{1}$, Joana M Esteves ${ }^{1}$, João Pedro Abreu ${ }^{1}$, Ana Francisca Pereira ${ }^{1}$, Maria Teresa Boncoraglio ${ }^{1}$, \\ Manuela Certo ${ }^{2}$, Rodolfo Abreu Ferreira ${ }^{3}$ \\ ${ }^{1}$ Internal Medicine Department, Hospital Santa Maria Maior, Barcelos, Portugal \\ ${ }^{2}$ Radiology Department, Hospital de Braga, Braga, Portugal \\ ${ }^{3}$ Vascular Surgery Department, Hospital de Braga, Braga, Portugal
}

\section{Doi: 10.12890/2019_001063 - European Journal of Case Reports in Internal Medicine - @ EFIM 2019}

Received: $10 / 02 / 2019$

Accepted: 26/02/2019

Published: 15/03/2019

How to cite this article: Leitão A, Esteves JM, Abreu JP, Pereira AP, Boncoragli TB, Certo M, Ferreira R. Deep venous thrombosis and a very rare finding: inferior vena cava infra-renal segment agenesis. EJCRIM 2019;6: doi:10.12890/2019_001063.

Conflicts of Interests: The Authors declare that there are no competing interests.

This article is licensed under a Commons Attribution Non-Commercial 4.0 License

\section{ABSTRACT}

Bilateral deep vein thrombosis (DVT) should prompt investigation for pro-thrombotic conditions and the exclusion of vascular compromise due to intrinsic and extrinsic factors. The authors present the case of a 47-year-old man admitted with bilateral DVT and diagnosed with inferior vena cava (IVC) infra-renal segment agenesis, and discuss the management of this rare condition.

\section{LEARNING POINTS}

- Bilateral deep vein thrombosis in young and otherwise healthy individuals should prompt investigation of the aetiology and risk factors in case life-long treatment and anticoagulation therapy is required.

- Although rare, anatomical variations and congenital malformations should be considered even in adult patients.

- Rare medical cases should prompt a multidisciplinary approach to investigation and treatment, as prognosis will depend on both therapeutic procedures and anticipation of complications.

\section{KEYWORDS}

Deep venous thrombosis, absence of inferior vena cava, inferior vena cava anomalies

\section{INTRODUCTION}

Congenital malformation and agenesis of the inferior vena cava (IVC) are extremely rare vascular anomalies, being present in only $0.0005-$ $1 \%$ of the general population ${ }^{[1]}$. The absence of the infra-renal segment of IVC seems to be the rarest anomaly ${ }^{[2,3]}$, with KILT syndrome (kidney anomaly, IVC anomaly and leg thrombosis) also beingvery uncommon ${ }^{[1]}$. Frequently, these are incidental findings on imaging studies but have been reported during abdominal surgery or when difficulties arise during femoral catheterization.

\section{CASE DESCRIPTION}

A 47-year-old man was admitted to our hospital with complaints of nausea, lumbar and lower limb pain and oedema, which had started the month before but had worsened during the previous week, to the point of rendering him unable to walk.

The patient reported no trauma, recent surgery, acute illness, recent immobilization, long-range flights or known triggers of his symptoms. His history included prostatitis at age 34 and elective cholecystectomy at age 37 . He reported no medication, alcohol, tobacco or recreational drug use. However, he did mention that his sister and her son have had DVT, that his nephew had been diagnosed with a factor V Leiden mutation, and that his family had never been formally studied. 
On physical examination, he appeared well, was alert, calm, normothermic and haemodynamically stable, and showed no signs of respiratory distress. He presented pain and bilateral erythematous pitting oedema up to the inguinal ligament, but no other relevant changes.

\section{METHODS}

Laboratory studies showed slight leucocytosis of 11,600 leucocytes $/ \mathrm{mm}^{3}$, with normal haemoglobin, platelet count, C-reactive protein, renal and hepatic markers, and prothrombin time, and an activated partial thromboplastin time within the reference values. Lower limb venous Doppler imaging showed DVT of the left common femoral vein with extension to both the left and right iliac veins. The patient was admitted for investigation and symptom control, and started on anticoagulation therapy with enoxaparin.

CT angiography of the thorax, abdomen and pelvis revealed absence of the IVC infra-renal segment and collateral venous drainage through the azygos system, and confirmed acute bilateral thrombosis of both the external and internal iliac veins (Figs. 1-3). No kidney lesions, masses, organomegaly, adenopathies or other alterations were seen.
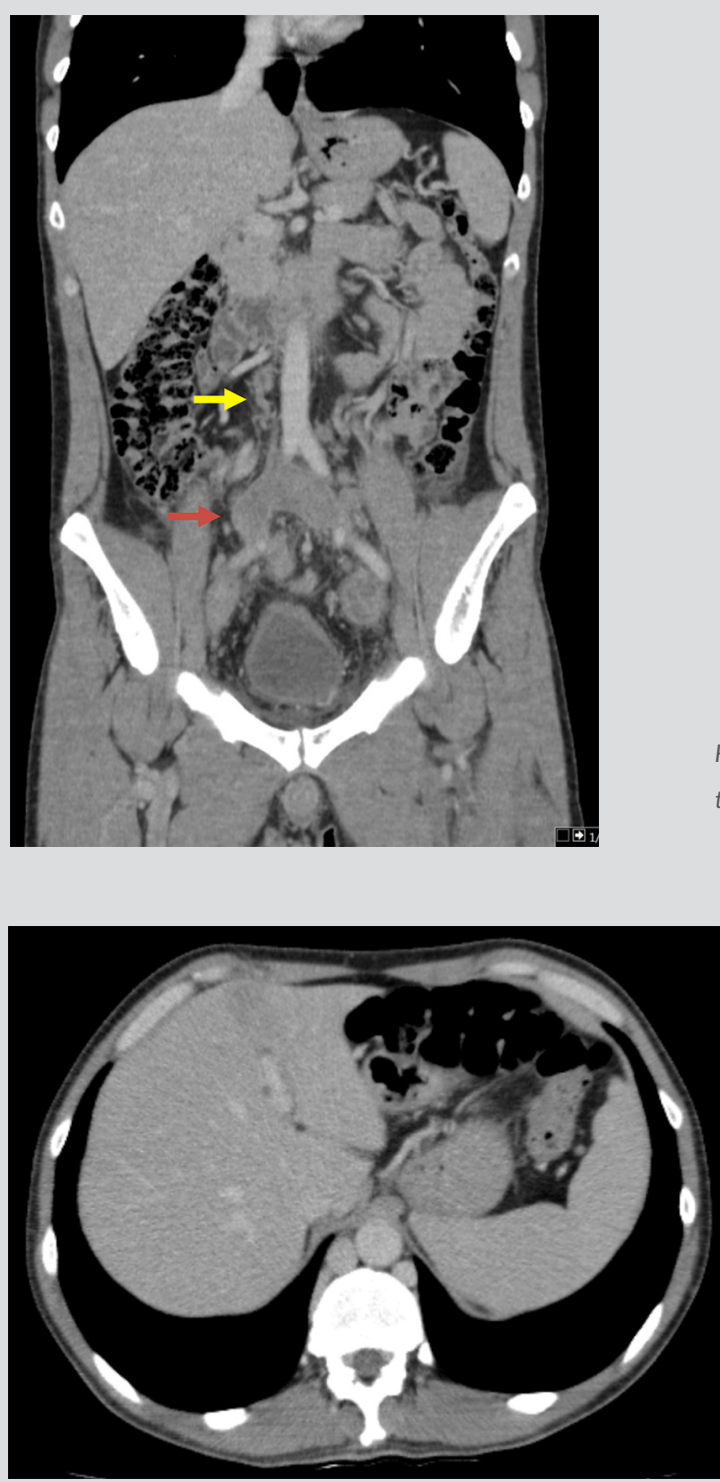

Figure 2. Contrast-enhanced axial CT scan showing collateral venous drainage through the azygos system
Figure 1. Contrast-enhanced coronal reformatted CT scan showing iliac vein thrombosis (red arrow) and absence of the inferior vena cava (yellow arrow)

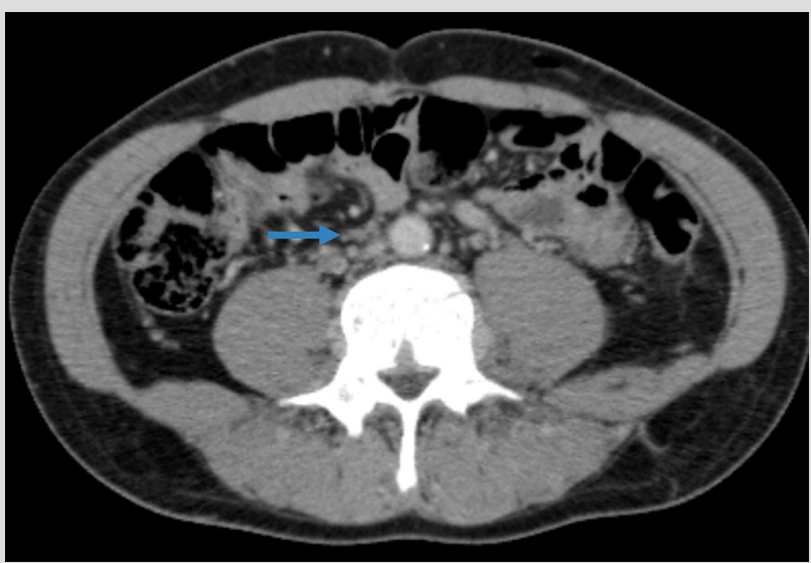

Figure 3.Ultrasound view of the external jugular vein thrombosis 
In light of the age and family history of the patient, a search for a prothrombotic condition that could have triggered the thrombosis was conducted, and included screening for subclinical infectious diseases (blood cultures and serologies for HIV, hepatitis B, C and syphilis were all negative), autoimmune diseases (negative for lupus and phospholipid syndrome antibodies) and indolent malignancies (upper endoscopy and colonoscopy, thyroid and testicular echography, free and total prostatic specific antigen dosing, beta-2-microglobulin, immunoglobulins and protein electrophoresis, the results of which were all normal).

Symptomatic control was achieved with anticoagulation, analgesics, and early but gradual ambulation, and the patient was discharged after 7 days.

After the acute phase had resolved, follow-up tests for protein S, C, ATIII and homocysteine levels were all normal. At 5-month follow-up, the lower limb complaints had been controlled with postural and compressive measures,while repeated imaging studies showed partial recanalization of the iliac veins.

Multidisciplinary team follow-up with the collaboration of immunohaemotherapy and vascular surgery specialists will be conducted in order to monitor the patient for symptomatic complications of post-thrombotic syndrome.

\section{DISCUSSION}

Highlighting of rare cases is essential so that future patients can be managed correctly. Patients with IVC anomalies can remain asymptomatic as long as there is functioning collateral venous drainage. However, when prothrombotic or venous insufficiency risk factors are present, symptoms may start to develop, usually with signs of venous insufficiency, pelvic congestion syndrome, DVT and compression syndromes from dilated collateral vascularization ${ }^{[4]}$.

Conservative therapy with anticoagulation, compression stockings and management of complications seem to be the mainstay of treatment, but invasive surgical interventions with an endovascular approach or even prosthetic replacement of the IVC have also been reported, with successful results ${ }^{[1,5]}$.

\section{REFERENCES}

1. Tufano A, Cannavacciuolo F, Gianno A, Cerbone A, Mangiacapra S, Coppola A, et al. Inferior vena cava agenesis and deep vein thrombosis in the young: a review of the literature and local experience. Semin Thromb Hemost 2017:43:827-835.

2. Milio G, Corrado E, Novo S, Licata G, Pinto A. Agenesis of the renal segment of inferior vena cava associated with venous stasis. Int Angiol 2010;29:385-388.

3. Kola MK, Sandeep S, Vankayalapati SH, Reddy MS. Absent infra-renal inferior vena cava presenting with varicose veins. J Assoc Physicians India 2017;65:105-106.

4. Menezes T, Haider EA, Al-Douri F, El-Khodary M, Al-Salmi I. Pelvic congestion syndrome due to agenesis of the infrarenal inferior vena cava. Radiol Case Rep 2019;14:36-40.

5. Sagban TA, Grotemeyer D, Balzer KM, Tekath B, Pillny M, Grabitz K, et al. Surgical treatment for agenesis of the vena cava: a single-centre experience in 15 cases. Eur J Vasc Endovasc Surg 2010;40:241-245. 